\title{
Coordinated Beamforming in Downlink CoMP Transmission System
}

\author{
Li Qiang, Yang Yang, Fang Shu, Wu Gang \\ National Key Laboratory Science and Technology on Communications \\ University of Electronic Science and Technology of China \\ Chengdu, Sichuan 610054, China \\ Email:\{liqiang1, yangyang, fangshu, wugang99\}@uestc.edu.cn
}

\begin{abstract}
Coordinated multi-point transmission/reception (CoMP) technique for 3GPP LTE-Advanced is to reduce the inter-cell interference (ICI) effectively, so as to enhance data rate/coverage and throughput for cell-edge users significantly. For downlink CoMP, most research work mainly focuses on joint processing/transmission (CoMP-JP) mode. However this mode needs the access points (AP) to share the UEs' data and channel state information (CSI) simultaneously, resulting in a high overhead on the network. Coordinated beamforming (CoMP-CBF), which can avoid the data information exchange is a better solution to reduce the overhead. In this paper, we propose a coordinated beamforming scheme in downlink CoMP transmission system. The proposed scheme exploits the signal leakage information to other cells to design the precoding vector to reduce the ICI. The simulation results indicate that the proposed scheme offers much higher performance gain compared with the CoMP-JP mode.
\end{abstract}

Keywords-LTE-A; CoMP; inter-cell interference; joint processing/transmission; coordinated beamforming

\section{INTRODUCTION}

Multiple-input multiple-output (MIMO) technique can effectively increase system peak spectrum efficiency in wireless communication and has been applied in 3GPP long term evolution (LTE). However, the cell-edge spectrum efficiency can't be promoted significantly because of the severe inter-cell interference (ICI). Therefore, In RAN1 \#53bis meeting a key promising technique called coordinated multi-point transmission and reception (CoMP) is added in the skeleton [1] for 3GPP LTE-Advanced (LTE-A) to solve this problem. Up to now, it's still under discussion. The CoMP technique can suppress the co-channel interference [2] from multi-cells, which also further improves the coverage and increases cell-edge/aggregate system throughputs. However, the existing problem of CoMP is the tradeoff between the performance and the feedback, signaling overhead, processing delays.

The coordinated multi-point system [3][4][5] can be seen as a single point system with additional $\mathrm{Tx} / \mathrm{Rx}$ antennas. One eNodeB contains one or multiple cells and each cell contains one or multiple access points (AP). The AP is a transmission and reception point, which is a physical node equipped with one or more antenna elements corresponding to one sector. One UE may be served by one or multiple APs from the same or different eNodeB simultaneously. Since the different eNodeB requires huge information exchange to implement coordination, it's agreed in RAN1 \#59bis meeting that coordination in the same eNodeB is supported.

To be specific, the downlink CoMP transmission is mainly divided into two categories: joint processing/transmission (CoMP-JP) and coordinated scheduling/beamforming (CoMP-CBF) [1-5].

In CoMP-JP mode as shown in Fig. 1, the data information to the UE is simultaneously transmitted from all APs under the control of the same eNodeB. The UE's data is distributed and jointly processed across the APs and the channel state information (CSI) is required for all the AP-UE pairs. Although CoMP-JP mode is incurring large system overhead, it can coherently or non-coherently improve the received signal quality and cancel actively interference for the UEs. The CoMP-JP mode can be used for serving one UE (SU) or multiple UEs (MU) using the given time/frequency resources at the same time.

Recently, most research work focuses on CoMP-JP mode. In [6][7], conventional zero-forcing (ZF) precoding scheme is applied in DL CoMP-JP. A related scheme is block diagonalization (BD) [8][9], which requires a limitation of the number of receive antennas and the number of data streams. And the precoding scheme in [6][7][9][10] based on signal-to-leakage-plus noise ration (SLNR) maximizes the sum of the achievable rate through minimizing the leakage to other UEs, which has no restriction as to the number of receive antennas per UE. Both DPC and THP (nonlinear precoding techniques) are considered in [11][12]. Though the nonlinear precoding can bring the best performance, it's difficult to implement in practice for the high complexity. Therefore, linear precoding in CoMP-JP is an optimized solution with relatively lower complexity requirements at both the APs and the UEs.

In the category of coordinated scheduling/beamforming as shown in Fig. 2, the data information to the UE is transmitted from its serving cell only but the cells in the CoMP set coordinate their transmission so that inter-cell interference can be reduced. Meanwhile, it is not necessary to share the UE's data information across multiple APs, 


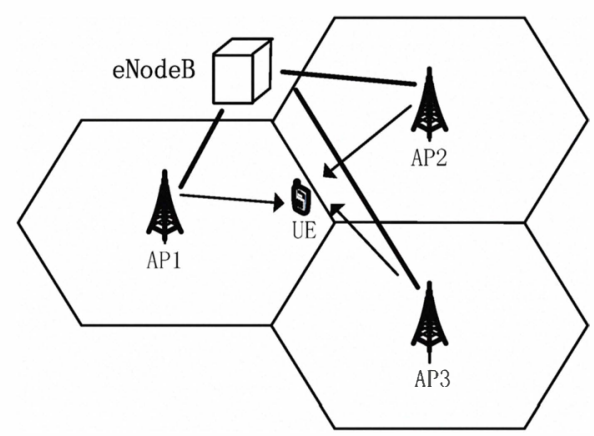

Fig. 1. CoMP-JP

which alleviates the heavy overhead on the network. To achieve the coordination, the UE needs to feed back information about the CSIs of the serving cell and the other cells in the CoMP set. CoMP-CBF mode can also increase the cell edge user throughput via interference management. Recent work on CoMP-CBF mode [9][13] has assumed implicit feedback which denotes the limited feedback at APs. Each UE feeds back the preferred precoding matrix indicator (PMI) for the serving cell. In addition to this, the eNodeB needs to know the PMIs that would result in minimum interference when they are used for transmission by other cells in CoMP set, and the eNodeB schedules corresponding APs to implement precoding. However, limited performance improvement arises from CoMP with limited-feedback, which in particular is suitable for FDD system.

So in this paper, we propose a coordinated beamforming scheme with explicit feedback which denotes the full channel state information. We can exploit the channel reciprocity to get this information in TDD system and use the feedback of explicit information in FDD system. The proposed scheme is based on the idea of SLNR [14] and the precoding metric takes the same form as SLNR. But the SLNR precoding is independently developed for the multiuser downlink channel in single cell environment. Unlike the precoding scheme in [14], the proposed scheme provides a solution for selecting a UE at each cell to maximize the total achievable rate, which considers not only the desired signal power in the serving cell but also the signal broadcasting to the other UEs in adjacent cells as interference. The simulation results will show that the proposed scheme outperforms zero-forcing precoding in CoMP-JP mode and is close to the optimal linear precoding in CoMP-JP.

The rest of the paper is organized as follows. Section II describes the system. In Section III, we present the coordinated beamforming scheme in downlink CoMP transmission system. Simulation results are presentd in Section IV, and conclusion are draw in Section V.

Throughout the paper, we use the following notation. $(\cdot)^{T}$ and $(\cdot)^{\dagger}$ denote transpose and conjugate transpose. $\operatorname{det}(\cdot)$ and $\operatorname{tr}(\cdot)$ denote determinant and trace of a matrix, respectively. $(\cdot)^{-1}$ denotes matrix inversion. $\|\cdot\|$ denotes

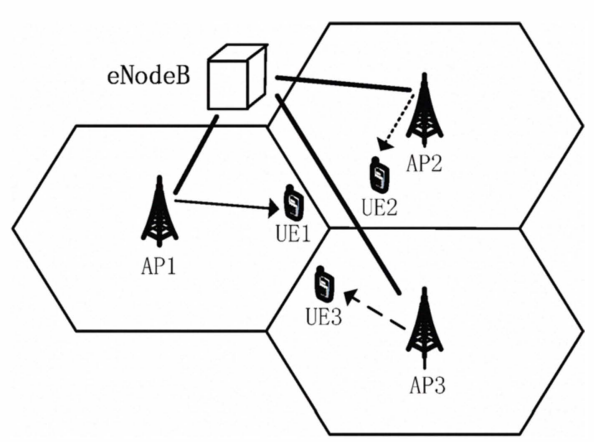

Fig. 2. CoMP-CBF

norm of a vector and $\|\cdot\|_{F}$ denotes Frobenius norm of a matrix. I represents the identity matrix.

\section{SYSTEM MODEL}

Considering the downlink CoMP-CBF mode comprised of $L$ cells in intra-eNodeB scenario, it is assumed that a single UE uniformly distributed at the edge of each cell is selected by the user scheduler of its serving cell. The UE and the AP are configured with $N_{r}$ receive antennas and $N_{t}$ transmit antennas, respectively. We restrict ourselves to one data stream per UE.

The signal received by user $k$ can be represented as

$$
\mathbf{y}_{k}=\mathbf{H}_{k}^{(k)} \mathbf{w}^{(k)} x_{k}+\sum_{i=1, i \neq k}^{L} \mathbf{H}_{k}^{(i)} \mathbf{w}^{(i)} x_{i}+\mathbf{n}_{k}
$$

where $\mathbf{H}_{k}^{(i)}$ denotes the $N_{r} \times N_{t}$ channel matrix from $i$-th cell ( $i$-th AP) to the $k$-th UE. $\mathbf{w}^{(i)}$ is a $N_{t} \times 1$ linear precoding vector in the $i$-th cell, $\left\|\mathbf{w}^{(i)}\right\|^{2}=1, \quad i=1, \cdots L . \mathbf{n}_{k}$ denotes the $N_{r} \times 1$ additive white Gaussian noise (AWGN) vector with zero mean and universal variance $\sigma^{2} . x_{i}$ is the signal data information for the $i$-UE and the total power constraint is imposed by $\mathbf{E}\left(\|x\|^{2}\right)=P_{t}$. The second term on the right hand side in (1) is the interference from the other adjacent cells. The signal-to-interference-plus-noise ratio (SINR) at the input of the receive of user $k$ is given by

$$
\operatorname{SINR}_{k}=\frac{P_{t}\left\|\mathbf{H}_{k}^{(k)} \mathbf{w}^{(k)}\right\|^{2}}{N_{0}+\sum_{i=1, i \neq k}^{L} P_{t}\left\|\mathbf{H}_{k}^{(i)} \mathbf{w}^{(i)}\right\|^{2}}
$$

where $N_{0}$ is the power of the noise. We assume that the UEs belonged to different cells in the CoMP set feed back the channel state information through reverse channel link under TDD mode or through feedback under FDD mode. All the APs share the channel state information under the control of the same eNodeB.

\section{COORDINATED BEAMFORMING SCHEME IN DOWNLINK COMP SYSTEM}

From the receive signal expression (1), the capacity of the $k$-th cell can be expressed as [15][16][17] 


$$
C^{(k)}=\log _{2} \operatorname{det}\left(\mathbf{I}+P_{t}\left(\mathbf{H}_{k}^{(k)} \mathbf{w}^{(k)}\right)\left(\mathbf{H}_{k}^{(k)} \mathbf{w}^{(k)}\right)^{\dagger}\left(\Psi^{(k)}\right)^{-1}\right)
$$

where $\boldsymbol{\Psi}^{(k)}$ is the covariance matrix of the noise and interference at the UE,

$$
\boldsymbol{\Psi}^{(k)}=\sum_{i=1, i \neq k}^{L} P_{t}\left(\mathbf{H}_{k}^{(i)} \mathbf{w}^{(i)}\right)\left(\mathbf{H}_{k}^{(i)} \mathbf{w}^{(i)}\right)^{\dagger}+N_{0} \mathbf{I}
$$

It's an optimization problem to find the precoding vectors for maximizing the total system capacity including the $L$ cells, which can be further denoted as

$$
\left(\mathbf{w}_{\text {opt }}^{(1)}, \ldots \mathbf{w}_{\text {opt }}^{(L)}\right)=\underset{\left(\mathbf{w}^{(1)}, \ldots \mathbf{w}^{(L)}\right)}{\arg \max }\left(\sum_{i=1}^{L} C^{(i)}\right)
$$

In order to simplify the optimization problem in (5), we take $L=2$ cells as an example to derive the coordinated beamforming vector. A block diagram of the system is shown in Fig. 3. We have referred to the derivation in [14][15][16].

$$
\begin{aligned}
& \left(\mathbf{w}_{\text {opt }}^{(1)}, \mathbf{w}_{\text {opt }}^{(2)}\right)=\underset{\left(\mathbf{w}^{(1)}, \mathbf{w}^{(2)}\right)}{\arg \max }\left(\sum_{i=1}^{2} C^{(i)}\right) \\
& =\underset{\left(\mathbf{w}^{(1)}, \mathbf{w}^{(2)}\right)}{\arg \max }\left(\log _{2}\left(1+\operatorname{SINR}^{(1)}\right)+\log _{2}\left(1+\operatorname{SINR}^{(2)}\right)\right)
\end{aligned}
$$

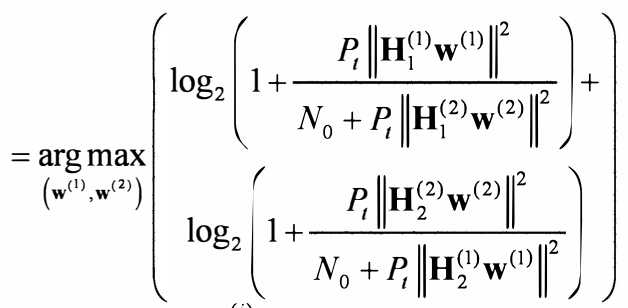

Assuming $\operatorname{SINR}^{(i)} \gg 1$, then we can make a close approximation that $\log _{2}\left(1+\operatorname{SINR}^{(i)}\right) \approx \log _{2}\left(\operatorname{SINR}^{(i)}\right), \mathrm{i}=1,2$. Expression (6) will turn into

$$
\begin{aligned}
& \left(\mathbf{w}_{\text {opt }}^{(1)}, \mathbf{w}_{\text {opt }}^{(2)}\right) \approx \underset{\left(\mathbf{w}^{(1)}, \mathbf{w}^{(2)}\right)}{\arg \max }\left(\log _{2}\left(\operatorname{SINR}^{(1)}\right)+\log _{2}\left(\operatorname{SINR}^{(2)}\right)\right) \\
& =\underset{\left(\mathbf{w}^{(1)}, \mathbf{w}^{(2)}\right)}{\operatorname{argmax}}\left(\begin{array}{c}
\log _{2}\left(\frac{P_{t}\left\|\mathbf{H}_{1}^{(1)} \mathbf{w}^{(1)}\right\|^{2}}{N_{0}+P_{t}\left\|\mathbf{H}_{1}^{(2)} \mathbf{w}^{(2)}\right\|^{2}}\right)+ \\
\log _{2}\left(\frac{P_{t}\left\|\mathbf{H}_{2}^{(2)} \mathbf{w}^{(2)}\right\|^{2}}{N_{0}+P_{t}\left\|\mathbf{H}_{2}^{(1)} \mathbf{w}^{(1)}\right\|^{2}}\right)
\end{array}\right) \\
& =\underset{\left(\mathbf{w}^{(1)}, \mathbf{w}^{(2)}\right)}{\arg \max }\left(\log _{2}\left(\frac{P_{t}\left\|\mathbf{H}_{1}^{(1)} \mathbf{w}^{(1)}\right\|^{2}}{N_{0}+P_{t}\left\|\mathbf{H}_{1}^{(2)} \mathbf{w}^{(2)}\right\|^{2}}\right)\left(\frac{P_{t}\left\|\mathbf{H}_{2}^{(2)} \mathbf{w}^{(2)}\right\|^{2}}{N_{0}+P_{t}\left\|\mathbf{H}_{2}^{(1)} \mathbf{w}^{(1)}\right\|^{2}}\right)\right) \\
& =\underset{\left(\mathbf{w}^{(1)}, \mathbf{w}^{(2)}\right)}{\arg \max }\left(\log _{2}\left(\frac{P_{t}\left\|\mathbf{H}_{1}^{(1)} \mathbf{w}^{(1)}\right\|^{2}}{N_{0}+\left\|\mathbf{H}_{2}^{(1)} \mathbf{w}^{(1)}\right\|^{2}}\right)\left(\frac{P_{t}\left\|\mathbf{H}_{2}^{(2)} \mathbf{w}^{(2)}\right\|^{2}}{N_{0}+P_{t}\left\|\mathbf{H}_{1}^{(2)} \mathbf{w}^{(2)}\right\|^{2}}\right)\right)
\end{aligned}
$$

Then we can get

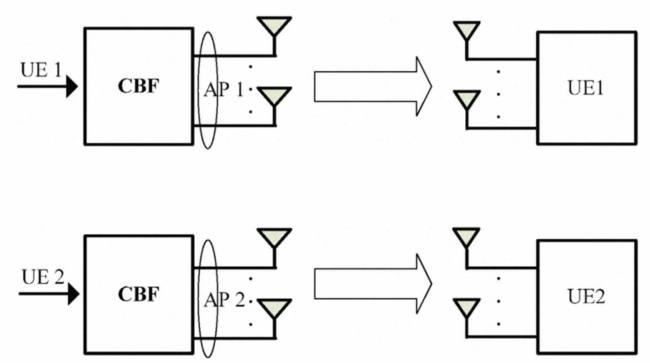

Fig. 3. Block diagram of coordinated beamforming in DL CoMP

$$
\begin{aligned}
& \mathbf{w}_{o p t}^{(1)}=\underset{\mathbf{w}^{(1)}}{\arg \max }\left(\frac{P_{t}\left\|\mathbf{H}_{1}^{(1)} \mathbf{w}^{(1)}\right\|^{2}}{N_{0}+P_{t}\left\|\mathbf{H}_{2}^{(1)} \mathbf{w}^{(1)}\right\|^{2}}\right) \\
& \mathbf{w}_{\text {opt }}^{(2)}=\underset{\mathbf{w}^{(2)}}{\arg \max }\left(\frac{P_{t}\left\|\mathbf{H}_{2}^{(2)} \mathbf{w}^{(2)}\right\|^{2}}{N_{0}+P_{t}\left\|\mathbf{H}_{1}^{(2)} \mathbf{w}^{(2)}\right\|^{2}}\right)
\end{aligned}
$$

Then expression (8) and (9) take the same form as SLNR metric, and the numerator denotes the signal to the serving $\mathrm{UE}$, the denominator refers to the noise plus interference caused by the signal intended for a desired UE on the other UEs in adjacent cells. So we can directly use the SLNR solution to get the optimal precoding vector.

$$
\mathbf{w}_{\text {opt }}^{(i)} \infty \text { max .eigenvector }\left(\left(N_{0} \mathbf{I}+\left(\tilde{\mathbf{H}}^{(i)}\right)^{\dagger} \tilde{\mathbf{H}}^{(i)}\right)^{-1}\left(\mathbf{H}^{(i)}\right)^{\dagger} \mathbf{H}^{(i)}\right)
$$

where

$$
\tilde{\mathbf{H}}^{(i)}=\left[\mathbf{H}_{1}^{(i)} \cdots \mathbf{H}_{i-1}^{(i)} \mathbf{H}_{i+1}^{(i)} \cdots \mathbf{H}_{L}^{(i)}\right]^{T},\left(L N_{r} \times N_{t}\right)
$$

It is shown that the optimal solution is given by (10) in terms of the eigenvector corresponding to the largest eigenvalue of the matrix $\left(N_{0} \mathbf{I}+\left(\tilde{\mathbf{H}}^{(i)}\right)^{\dagger} \tilde{\mathbf{H}}^{(i)}\right)^{-1}\left(\mathbf{H}^{(i)}\right)^{\dagger} \mathbf{H}^{(i)}$. The norm of $\mathbf{w}_{\text {opt }}^{(i)}$ is adjusted to $\left\|\mathbf{w}_{\text {opt }}^{(i)}\right\|^{2}=1$. Although this scheme is derived under the case $L=2$, it can be extended to arbitrary number of cells by adding the channels between the serving cell and the UEs in other cells in (11). The proposed scheme in (10) is sub-optimal in terms of SINR maximization, yet simple enough that one can find the best linear precoding solution, which has the same identity with SLNR scheme.

For CoMP-CBF mode, some schemes with limited feedback are under discussion. As mentioned before, the UE estimates the channel matrices and finds the best-matching precoder from the codebook known to both the AP and UE. Then the UE feeds back the best PMI index of the serving cell and the least interfering PMI of the neighboring cell to implement coordination. By PMI feedback, beam collision can be avoided. However, the performance is limited for implicit feedback. And it is complex for the eNodeB to schedule different APs to choose the proper PMIs when the number of APs is increasing. Therefore, it still needs a lot of work to do. 


\section{Simulation Results}

In this section, we present the initial simulation results to illustrate the performance of proposed scheme. We consider that each cell has selected one UE with the $L=2$ APs' coordination. The detailed simulation parameters are listed in Table I.

Fig. 4 shows the cumulative distribution function (CDF) results of the sum system capacity. Each AP is configured with $N_{t}=2$ antennas. As a reference, we give the nonCoMP scheme that means there is no coordination among APs and each UE will suffer from severe interference from other cells. The CoMP-JP scheme uses zero-forcing (ZF) or singular value decomposition (SVD) precoding transmitting two data streams to a single UE with equal power allocation, which is across two APs' coordination under the control of the same eNodeB.

From the results we can see that the CoMP-JP mode or the CoMP-CBF mode both are better than Non-CoMP scheme, which confirms that the CoMP technique is a very promising scheme to improve the cell edge capacity. The proposed scheme outperforms the CoMP-JP ZF scheme and it is close to the CoMP-JP SVD scheme. In CoMP-JP mode, all APs coordinates together to serve the UE, so the interference from other cell in the CoMP set turns into the desired useful signal. SVD is an optimal linear precoding scheme for the single UE when it applies in CoMP-JP mode. So our proposed scheme is close to the optimal scheme, however it yields better performance than CoMP-JP ZF scheme. It can be understood that the proposed scheme based on the ideal of SLNR which takes noise level into account differing from the ZF scheme. It's a sub-optimal solution in terms of the SINR maximization solution. Compared with CoMP-JP mode, the CoMP-CBF mode only requires the channel information exchange which reduces a heavy overhead on the network.

TABLE I

SIMULATION PARAMETERS

\begin{tabular}{|c|c|}
\hline Parameters & Value \\
\hline Layout & $\begin{array}{c}\text { Hexagonal grid, 3 cells sites, } 3 \\
\text { sectors per site }\end{array}$ \\
\hline Inter-site distance & $500 \mathrm{~m}$ \\
\hline Channel model & SCM, urban macro, 1 path \\
\hline Users per cell & 1, dropped uniformly \\
\hline $\begin{array}{c}\text { Minimum distance between UE } \\
\text { and cell }\end{array}$ & $>=35$ metres \\
\hline Number of antennas $\left(N_{t}, N_{r}\right)$ & $(4 / 2,2)$ \\
\hline $\begin{array}{c}\text { Antennas separation in } \\
\text { wavelength }\left(N_{t}, N_{r}\right)\end{array}$ & $(10,0.5)$ \\
\hline $\begin{array}{c}\text { Distance-dependent path loss } \\
\text { Shadowing standard deviation }\end{array}$ & L=34.5+35log10(R), R in \\
\hline mandwidth & $8 \mathrm{~dB}$ \\
\hline Carrier frequency & $10 \mathrm{MHz}$ \\
\hline UE speed & $2 \mathrm{GHz}$ \\
\hline Receiver processing & $3 \mathrm{~km} / \mathrm{h}$ \\
\hline Channel estimation error & $\mathrm{MMSE}$ \\
\hline AP transmit power & Ideal estimation \\
\hline Noise density & $46 \mathrm{dBm}$ \\
\hline
\end{tabular}

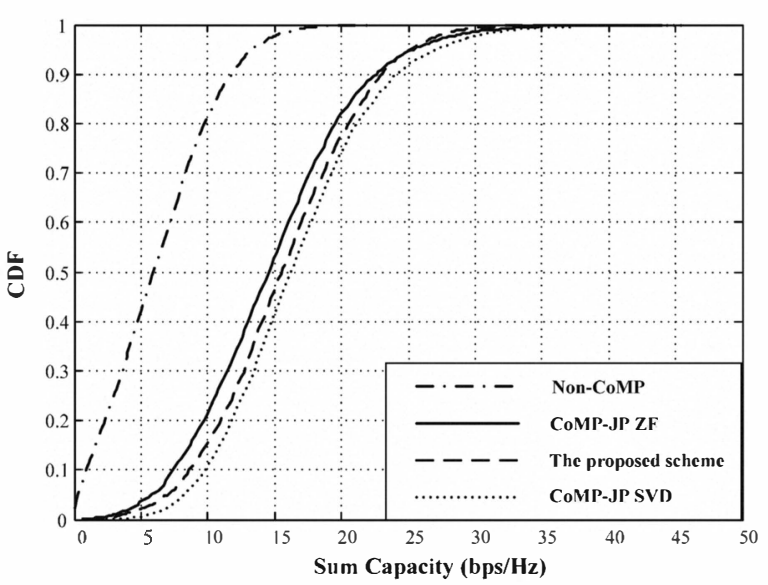

Fig. 4. CDF of system sum capacity

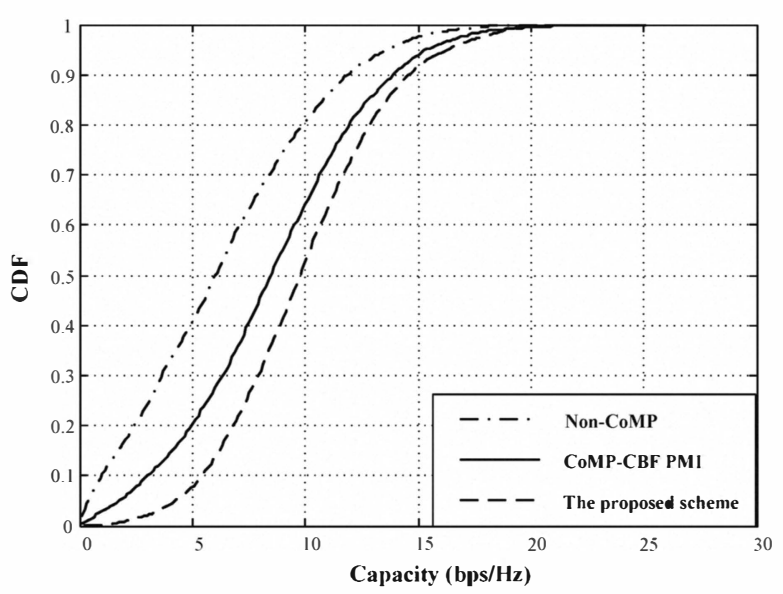

Fig. 5. CDF of capacity in one cell

In Fig. 5, the CDF results of the capacity in one cell are given. In this case, we choose one UE which denotes the capacity of its cell to analyze the performance gain, each AP has $N_{t}=4$ antennas. The codebook is the DFT codebook with the size of $(16,4)$, the quantization vectors are 16 and each vector has a dimension of 4 . The UE feeds back $B=4$ bits index PMI. Each AP sends only one data stream to one UE in all schemes. Because of the severe interference without coordination, Non-CoMP scheme performs worst. It can be seen that the proposed scheme performs much better than the CoMP-CBF scheme with PMIs feedback and shows an obvious gain. Since the proposed scheme directly exploits the explicit channel information to achieve beamforming and takes the noise level into consideration.

\section{CONCLUSION}

In this paper, we have proposed a coordinated beamforming scheme in downlink CoMP system. For CoMP-JP mode, it needs UEs' data and channel information to exchange across APs which results in a large overhead. The proposed scheme has only required the channel state information to further reduce the overhead on the network 
and has provided a considerable performance. The proposed scheme has utilized the signal leakage information to other cells to design the precoding vector to mitigate the multi-cell interference. The proposed scheme can be adopted into practice system by SRS based feedback under TDD mode. The simulation results have shown that the proposed scheme shows a much higher performance gain even outperforms zero-forcing scheme in CoMP-JP mode. The CoMP-CBF mode has started to attract much attention for future research work because of the low overhead and good performance. In general, CoMP-CBF mode is the trend for CoMP technique to be deployed into practice, which can meets the requirements set by LTE-A.

\section{ACKNOWLEDGMENT}

The project was supported by the Key Laboratory of Universal Wireless Communications Lab (Beijing University of Posts and Telecommunications), Ministry of Education, China and supported in part by Chinese Important National Science \& Technology Projects under Grant (2009ZX03003009, 2009ZX03004-001), National Science and Technology Ministry (ID: 2008BAH30B12).

The project was also supported by the National Natural Science Foundation of China $(60702073,60972029)$.

\section{REFERENCES}

[1] 3GPP TR36.913, "Requirements for Further Advancements for EUTRA(LTE-Advanced)(Release 8)," 3GPP, www.3gpp.org, Jun,2008.

[2] 3GPP, R1-083569, "Further discussion on Inter-Cell Interference Mitigation through Limited Coordination," Samsung, Sep. 29-Oct. 3, 2008 .

[3] 3GPP, R1-083686, "Views on Coordinated Multipoint Transmission/Reception in LTE-Advanced," NTT DOCoMo, Sep. 29-Oct. 3, 2008.
[4] 3GPP, R1-082469, "LTE-Advanced-Coordinated Multipoint Transmission/reception," Ericsson, Jun. 30-Jul. 4, 2008.

[5] 3GPP, R1-083049, "Consideration on CoMP for LTE-Advanced," Hua Wei, Aug. 18-22, 2008.

[6] 3GPP, R1-090028, "Leakage-based precoding for CoMP in LTE-A," Mitsubishi Electric, Jan. 12-16, 2009.

[7] H. Zhang, N. B. Mehta, A. F. Molisch, J. Zhang, and H. Dai, "Asynchronous Interference mitigation in Cooperative Base Station Systems," IEEE Trans. Wireless Commun., vol. 7, no. 1, pp. 155-164, Jan. 2008.

[8] 3GPP, R1-093273, "SRS feedback mechanism based CoMP schemes in TD-LTE-Advanced," CMCC, Aug. 24-28, 2009.

[9] 3GPP, R1-092585, "Analysis of Feedback Mechanisms for CoMP," InterDigital, Jun. 29-Jul. 3, 2009

[10] 3GPP, R1-093961, "Feedback Compression Methods and Their Performance Comparison," Motorola, Oct. 12-16, 2009.

[11] 3GPP, R1-090246, "An Efficient DPC MIMO Scheme for Downlink CoMP in LTE-A," Hitachi, Jan. 12-16, 2009.

[12] 3GPP, R1-091180, "Further Considerations for Downlink CoMP Transmission using DPC MIMO," Hitachi, Mar. 23-27, 2009.

[13] 3GPP, R1-093343, "Comparison of CSI Feedback Schemes," AlcatelLucent, Aug. 24-28, 2009.

[14] M. Sadek, A. Tarighat and A.H. Sayed, "A Leakage-Based Precoding Scheme for Downlink Multi-User MIMO Channels," IEEE Trans. Wireless Commun., vol. 6, no. 5, pp. 1-12, May. 2007.

[15] B. O. Lee, H. W. Je, O. Shin and K. B. Lee, "A Novel Uplink MIMO Transmission Scheme in a Multicell Environment," IEEE Trans. Wireless Commun., vol. 8, no. 10, pp. 4981-4987, Oct. 2009.

[16] Y. Wu, J. Zhang, M. Xu, S. Zhou, and X. Xu, "Multiuser MIMO downlink precoder design based on the maximal SJNR criterion," in Proc. IEEE Global Commun. Conf. 2005, St. Louis, MO, pp. 26942698.

[17] T. M. Cover and J. A. Thomas, Elements of Information Theory, New York, John Wiley \& Sons, 1991. 